COMMUNICATIONS IN

ANALYSIS AND GEOMETRY

Volume 12, Number 1, 111-141, 2004

\title{
Kähler-Ricci Flow and the Poincaré-Lelong Equation
}

\author{
LEI NI ${ }^{1}$ AND LUEN-FAI TAM ${ }^{2}$
}

\section{Introduction.}

In [M-S-Y], Mok-Siu-Yau studied complete Kähler manifolds with nonnegative holomorphic bisectional curvature by solving the Poincaré-Lelong equation

$$
\sqrt{-1} \partial \bar{\partial} u=\operatorname{Ric}
$$

where Ric is the Ricci form of the manifold. In [M-S-Y], the authors solved (0.1) under the assumptions that the manifold is of maximal volume growth and the scalar curvature decays quadratically. On the other hand, in a series of papers of W.-X. Shi [Sh2-4], Kähler-Ricci flow

$$
\frac{\partial}{\partial t} g_{\alpha \bar{\beta}}=-R_{\alpha \bar{\beta}}
$$

has been studied extensively and important applications were given. In [N1] and [N-S-T], the Poincaré-Lelong equation has been solved under more general conditions than in $[\mathrm{M}-\mathrm{S}-\mathrm{Y}]$. The conditions in $[\mathrm{N}-\mathrm{S}-\mathrm{T}]$ are more in line with the conditions in [Sh2-4]. Since a solution of $(0.1)$ is a potential for the Ricci tensor, it is interesting to see if one can apply (0.1) to study solutions of $(0.2)$.

In this work, on the one hand we shall study the Kähler-Ricci flows by using solutions of the Poincaré-Lelong equation. On the other hand, we will also refine some of the results in [Sh3, C-Z, C-T-Z] and give new applications. The hinge between the equations $(0.1)$ and $(0.2)$ is that by solving (0.1) one can then construct a function $u(x, t)$ which satisfies the time-dependent heat equation $\left(\frac{\partial}{\partial t}-\Delta\right) u(x, t)=0$ and the time-dependent Poincaré-Lelong equation $\sqrt{-1} \partial \partial \partial=\operatorname{Ric}_{g(t)}$ simultaneously. It then can simplify the study of $(0.2)$ quite a bit. It also suggests some of the refined

\footnotetext{
${ }^{1}$ Research partially supported by NSF grant DMS 0196405 and DMS-0203023, USA.

${ }^{2}$ Research partially supported by Earmarked Grant of Hong Kong \#CUHK4217/99P.
} 
estimates in the second part of this paper. We should point out here that the simplification in this paper is that $|\nabla u|^{2}$ helps to obtain a sharp uniform curvature estimates (Cf. Theorem 1.3), which holds as an equality for the Kähler-Ricci soliton. It is different from the compact case as in [Co1], where one restricts the deformation of the metric within a fixed cohomology class and can then appeal to Yau's solution to the Monge-Amperé equation by reducing (0.2) to a single equation.

Let $\left(M^{m}, g_{\alpha \bar{\beta}}(x)\right)$ be a complete noncompact Kähler manifold with bounded and nonnegative holomorphic bisectional curvature. Let $\mathcal{R}_{0}$ be the scalar curvature of $M$. In [Sh3], it was proved that (0.2) has long time solution with initial metric $g_{\alpha \bar{\beta}}(x)$ satisfying the assumption that

$$
k(x, r) \leq C(1+r)^{-\theta}
$$

for some constants $C$ and $\theta>0$ for all $x$ and $r$. Here $k(x, r)$ denotes the average of $\mathcal{R}_{0}$ on $B(x, r)$, the geodesic ball of radius $r$ with center at $x$. The idea of the proof of the long time existence in [Sh3] is to use the parabolic version of the third derivative estimate for the Monge-Amperé equation together with a careful estimate of the volume element. The computation is rather tedious. In this work, we will use the solution to (0.1) constructed in [N-S-T] (more precisely the uniform curvature estimate (1.24) in Theorem 1.3) to give an alternate (and much simpler, we believe) proof for the long time existence under the assumption that

$$
\int_{0}^{\infty} k(x, r) d r \leq C
$$

for some $C$ independent of $x$. Our proof uses a maximum principle which is a generalization of that in $[\mathrm{K}-\mathrm{L}]$, and an idea similar to those in $[\mathrm{Cw}]$. Our assumption here is different from but somewhat stronger than Shi's (0.3). However it has covered the interesting cases in [Sh2-3], namely the cases $k(x, r) \leq C(1+r)^{-1-\delta}$, on which interesting geometric results could be obtained. On the other hand we also can prove a long time existence result under a more flexible condition. Namely, we show that there exists long time solution to $(0.2)$ if

$$
k(x, r) \leq \epsilon(r)
$$

for all $x$ (with some fixed function $\epsilon(r)$ ) with $\epsilon(r) \rightarrow 0$ as $r \rightarrow \infty$. Recently in [C-T-Z], it is proved that if the complex dimension of $M$ is $m=2$ and $M$ has maximal volume growth, then (0.2) has long time solution if (0.5) 
holds for some $x$ and for some function $\epsilon(r)$ which tends to zero as $r \rightarrow$ $\infty$. The proof there is an indirect blow-up argument. It also used some special features in dimension 2, such as the Guass-Bonnet formula for the four dimensional Riemannian manifolds. In order to prove the long time existence under the assumption (0.5), we need a more precise estimate for the volume element $F(x, t)=\log \left[\operatorname{det}\left(g_{\alpha \bar{\beta}}(x, t)\right) / \operatorname{det}\left(g_{\alpha \bar{\beta}}(x, 0)\right)\right]$, where $g_{\alpha \bar{\beta}}(x, t)$ is the solution of (0.2). In fact, we prove the following results, see Theorem 2.1 and Corollary 2.1:

Theorem. Suppose (0.2) has a solution on $M \times[0, T)$. Then we have the following:

(a) There exists a constant $C>0$ depending only on $m$ such that for $0<t<T$,

$$
-F\left(x_{0}, t\right) \geq C \int_{0}^{\sqrt{t}} s k\left(x_{0}, s\right) d s .
$$

(b) If in addition, $k(x, r) \leq k(r)$ for some function $k(r)$ for all $x$, then

$$
-\mathfrak{m}(t) \leq C^{\prime} \int_{0}^{R} s k(s) d s
$$

where $R^{2}=a t(1-\mathfrak{m}(t)), C$ and $a$ are constants depending only on $m$. Here $\mathfrak{m}(t)=\inf _{x \in M} F(x, t)$.

From the two-sidedness of the above estimates on $F(x, t)$ one can see that they are almost optimal. By comparing with previous estimates obtained in $[\mathrm{N}-\mathrm{S}-\mathrm{T}]$ and [N2] for the Poisson equation and the linear heat equation, the refined estimates here are sharp in certain cases and fit into the theory for the linear equation. The above mentioned estimates will be proved by using, the by-now standard estimates on the heat kernels of Li-Yau in [L-Y]. There is no need to construct special exhaustion functions as in [Sh2-3, $\mathrm{C}-\mathrm{Z}, \mathrm{C}-\mathrm{T}-\mathrm{Z}]$. As a consequence, a gap theorem, which is a little bit more general than those in $[\mathrm{C}-\mathrm{Z}]$, is obtained, see Corollary 2.3. In particular, we show that any bounded solution to the Poisson equation $\Delta u=\mathcal{R}_{0}(x)$ is a constant, provided $M$ has bounded nonnegative bisectional curvature. In other words, if $M$ is nonflat, $\Delta u=\mathcal{R}_{0}(x)$ has no bounded solution. This answers a question asked by R. Hamilton. Namely, solving Poisson for $\mathcal{R}_{0}(x)$ is different from arbitrary $f(x)$ since one can easily construct bounded solution to $\Delta u=f(x)$ for nonzero compact supportted $f(x)$. This is also related to the gradient estimates of Chow in $[\mathrm{Cw}]$. In $[\mathrm{Y}]$, it was proved that, on a complete Riemannian manifold with nonnegative Ricci curvature, any negative (positive) harmonic function is a constant. We prove that a similar 
result holds for $\Delta u=\mathcal{R}_{0}(x)$. Namely, $\Delta u=\mathcal{R}_{0}(x)$ has no nonconstant negative solution, provided $M$ has bounded nonnegative bisectional curvature and (0.2) has long time solution.

When $\left(M, g_{\alpha \bar{\beta}}(x, 0)\right)$ has the maximum volume growth, using the estimates mentioned above the results in [C-Z] on the Steinness and the topology of $M$ can be refined. Namely we show that if $\left(M, g_{\alpha \bar{\beta}}(x, 0)\right)$ is of maximum volume growth and $\int_{0}^{r} s k(x, s) d s \leq \phi(r)$ with $\phi(r) \rightarrow 0$ as $r \rightarrow \infty, M$ is Stein and diffeomorphic to $\mathbb{R}^{2 m}$ for $m \geq 3$, homeomorphic to $\mathbb{R}^{4}$ for $m=2$.

Another application of the estimates of $F$ and $(0.1)$ is that one can prove the preservation of the decay rate of $\mathcal{R}_{0}$ in a certain sense. For example, we will prove in Theorem 2.3 that if $\int_{0}^{r} s k(x, s) d s \leq C \log (1+r)($ or $C(1+r))$, where $k(x, r)$ is the average of the scalar curvature at $t=0$, then we still have $\int_{0}^{r} s k_{t}(x, s) d s \leq C^{\prime} \log (1+r)\left(C^{\prime}(1+r)\right.$, respectively), where $k_{t}(x, r)$ is the average of the scalar curvature at time $t$. Note that the constant $C^{\prime}$ is independent of $t$. This might be useful in analyzing the singularity models obtained by the blow-up procedure as in [H3].

From the methods of proof of the estimates of $F$, we can show that, under a rather weak decay condition on $\mathcal{R}_{0}$, the volume growth is preserved in the sense that for any $t>0$,

$$
\lim _{r \rightarrow \infty} \frac{V_{t}(o, r)}{V_{0}(o, r)}=1
$$

where $V_{t}(o, r)$ is the volume of the geodesic ball with center at $o$ and radius $r$ with respect to $g_{\alpha \bar{\beta}}(x, t)$. This generalizes the results of [H3, Sh2, C-Z, C-T-Z].

In [Sh2], under the assumption that $\theta=2$ in (0.3) and that $M$ has positive holomorphic bisectional curvature, Shi proved that the rescaled metric $\widehat{g}_{\alpha \bar{\beta}}(x, t)=g_{\alpha \bar{\beta}}(x, t) / g_{v \bar{v}}\left(x_{0}, t\right)$ subconverges to a flat Kähler metric on $M$, where $x_{0}$ is a fixed point and $v$ is a fixed nonzero $(1,0)$ vector at $x_{0}{ }^{3}$. If $M$ has maximal volume growth and if the limit metric is complete, then one can conclude that $M$ is biholomorphic to $\mathbb{C}^{m}$. It is pointed out in [C-Z] that from [Sh2] it is unclear why the property of completeness is true. In Proposition 3.1, we will prove that if the scalar curvature $\mathcal{R}_{0}$ has pointwise quadratic decay, then the largest eigenvalue of the limit metric with respect to the initial metric grows at least like $r_{0}^{a}(x)$ for some $a>0$, where $r_{0}(x)$ is the distance function to a fixed point with respect to the initial metric. This is a consequence of the result that volume elements of the rescaled metrics

\footnotetext{
${ }^{3}$ Added in proof: There is a gap in the proof of the convergence in [Sh2]. See [C-T] for details.
} 
converge to the solution of the Poincaré-Lelong equation constructed in [N$\mathrm{S}-\mathrm{T}$ ], see Theorem 3.1. We believe that this new piece of information will be helpful in studying the completeness of the limiting metric.

Here is how we organize this paper. In $\S 1$, we will give an alternate proof of long time existence for $(0.2)$. In $\S 2$, we will give more refined estimates for $F(x, t)$ together with some applications. In $\S 3$, we will study the asymptotic behavior of $F(x, t)$.

We shall use the differential inequalities for Kähler-Ricci flow of Cao [Co2-3] from time to time, which is also called Harnack inequality for the Ricci flow (Cf. [H4]) since it implies a Harnack type estimate. Since this and similar results originate from the fundamental work of Li-Yau [L-Y] and Hamilton [H4], it seems to be more appropriate to call them Li-Yau-Hamilton type inequalities. We shall adopt this terminology in this work.

The second author would like to thank Shing-Tung Yau for useful conversations.

\section{Long time existence via Poincaré-Lelong equation.}

Let $\left(M^{m}, g_{\alpha \bar{\beta}}(x)\right)$ be a complete noncompact Kähler manifold with bounded nonnegative holomorphic bisectional curvature. Consider the Kähler-Ricci flow:

$$
\frac{\partial}{\partial t} g_{\alpha \bar{\beta}}(x, t)=-R_{\alpha \bar{\beta}}(x, t)
$$

such that $g_{\alpha \bar{\beta}}(x, 0)=g_{\alpha \bar{\beta}}(x)$.

In [Sh1-3], short time existence of (1.1) was established, and the long time existence was also proved under the assumption that

$$
f_{B(x, r)} \mathcal{R}_{0} d V \leq C r^{-\theta}
$$

for some constants $C$ and $\theta>0$ for all $x$ and $r$. Here $\mathcal{R}_{0}$ is the scalar curvature of the initial metric and $f_{B_{x}(r)} \mathcal{R}_{0} d V$ is the average of $\mathcal{R}_{0}$ on the geodesic ball $B(x, r)$ with center at $x$ and radius $r$. The proof of the long time existence in [Sh2, Sh3] is rather complicated. In this section, with the help of solutions of the Poincaré-Lelong equation we shall give a simple proof of the long time existence by using a maximum principle. Our assumption on $\mathcal{R}_{0}$ is a little bit different from (1.2).

Let us recall the result on short time existence of Shi [Sh3].

Theorem 1.1. Let $\left(M^{m}, g_{\alpha \bar{\beta}}(x)\right)$ be a complete noncompact Kähler manifold with nonnegative holomorphic bisectional curvature such that the scalar 
curvature $\mathcal{R}_{0}$ is bounded by $C_{0}$. Then (1.1) has a solution on $M \times[0, T)$ for some $T>0$ depending only $m$ and $C_{0}$ such that the following are true.

(i) $\left(M, g_{\alpha \bar{\beta}}(x, t)\right)$ is a Kähler metric with nonnegative holomorphic bisectional curvature for $0 \leq t<T$.

(ii) There exists $C>0$ such that

$$
C^{-1} g_{\alpha \bar{\beta}}(x, 0) \leq g_{\alpha \bar{\beta}}(x, t) \leq g_{\alpha \bar{\beta}}(x, 0),
$$

and

$$
0 \leq \mathcal{R}(x, t) \leq C
$$

for all $(x, t) \in M \times[0, T)$.

Before we give our proof on the long time existence, let us fix the notations. For any smooth function $f$, let $\Delta f=g^{\alpha \bar{\beta}}(x, t) \frac{\partial^{2} f}{\partial z^{\alpha} \partial \bar{z}^{\beta}},|\nabla f|^{2}=$ $g^{\alpha \bar{\beta}}(x, t) f_{\alpha} f_{\bar{\beta}}$. Summation convention is understood. We also use $\tilde{\Delta}$ and $\widetilde{\nabla}$ to denote the Laplacian and the gradient with respect to a fixed metric $g_{\alpha \bar{\beta}}(x)$ or the initial metric $g_{\alpha \bar{\beta}}(x, 0)$ of the solution of $(1.1) . B_{t}(x, r)$ is the geodesic ball of radius $r$ with respect to the metric $g_{\alpha \bar{\beta}}(x, t)$ and $V_{t}(x, r)$ be the volume of $B_{t}(x, r)$ with respect to $g_{\alpha \bar{\beta}}(x, t)$. We may also use the ones without $t$ to denote the balls and volumes for a fixed metric. The same convention applies to the distance function $r_{t}(x, y)$ between two points $x, y \in M$ as well as the volume element $d V_{t}$. As in [Sh2], throughout this work, let

$$
F(x, t)=\log \left(\frac{\operatorname{det}\left(g_{\alpha \bar{\beta}}(x, t)\right)}{\operatorname{det}\left(g_{\alpha \bar{\beta}}(x, 0)\right)}\right) .
$$

Then for the solution of (1.1)

$$
\begin{gathered}
d V_{t}=e^{F} d V, \\
F(x, t)=-\int_{0}^{t} \mathcal{R}(x, \tau) d \tau
\end{gathered}
$$

where $\mathcal{R}(x, t)$ is the scalar curvature of the metric $g_{\alpha \bar{\beta}}(x, t)$. For the solution of (1.1), we have the following maximum principle, which is of independent interest. The proof follows the idea in [K-L] (see also Li's lecture notes [Li]).

Let $g_{i j}(x, t)$ be a smooth family of complete Riemannian metrics defined on $M$ with $0 \leq t \leq T_{1}$ for some $T_{1}>0$ satisfying the following properties: There exists a constant $C_{1}>0$ such that for any $T_{1} \geq t_{2} \geq t_{1} \geq 0$

$$
C_{1} g_{i j}\left(x, t_{1}\right) \leq g_{i j}\left(x, t_{2}\right) \leq g_{i j}\left(x, t_{1}\right)
$$

for all $x \in M$. 
Theorem 1.2. With the above assumptions and notations, let $f(x, t)$ be a smooth function such that $\left(\Delta-\frac{\partial}{\partial t}\right) f(x, t) \geq 0$ whenever $f(x, t) \geq 0$. Assume that

$$
\int_{0}^{T_{1}} \int_{M} \exp \left(-a r_{0}^{2}(x)\right) f_{+}^{2}(x, s) d V_{0} d s<\infty
$$

for some $a>0$, where $r_{0}(x)$ is the distance function to a fixed point $o \in M$ with respect to $g_{i j}(x, 0)$. Suppose $f(x, 0) \leq 0$ for all $x \in M$. Then $f(x, t) \leq 0$ for all $(x, t) \in M \times\left[0, T_{1}\right]$.

Proof. Let $F(x, t)$ be such that $d V_{t}=e^{F}(x, t) d V_{0}$. By (1.7), we have

$$
\frac{\partial}{\partial t} F \leq 0
$$

Let $0<T \leq T_{1}$ which will be specified later and let

$$
g(x, t)=\frac{-r_{T}^{2}(x)}{4(2 T-t)}, \quad \text { on } M \times[0, T] .
$$

Here $r_{T}(x)$ is the distance function to $o \in M$ with respect to $g_{\alpha \bar{\beta}}(x, T)$. It is easy to check that

$$
\left|\nabla_{T} g\right|^{2}+\frac{\partial g}{\partial t}=0
$$

Here $\nabla_{T}$ is the gradient with respect to $g_{\alpha \bar{\beta}}(x, T)$. By $(1.7), g_{i j}$ is nonincreasing in $t$, hence we have

$$
|\nabla g|^{2}+\frac{\partial g}{\partial t} \leq\left|\nabla_{T} g\right|^{2}+\frac{\partial g}{\partial t}=0
$$

for $t \in[0, T]$. Let $\varphi(x)$ be a cut-off function which we will specify later. We have

$$
\begin{aligned}
0 & \leq \int_{0}^{T} \int_{M} \varphi^{2} e^{g} f_{+}\left(\Delta-\frac{\partial}{\partial t}\right) f d V_{s} d s \\
& =\int_{0}^{T} \int_{M} \varphi^{2} e^{g} f_{+}(\Delta f) d V_{s} d s-\frac{1}{2} \int_{0}^{T} \int_{M} \varphi^{2} e^{g} \frac{\partial}{\partial t}\left(f_{+}^{2}\right) d V_{s} d s
\end{aligned}
$$

Here $f_{+}:=\max \{0, f\}$. Now we calculate the last two terms in the above 
inequality.

$$
\begin{aligned}
\int_{M} \varphi^{2} e^{g} f_{+}(\Delta f) d V_{s}= & -\int_{M} \varphi^{2} e^{g}\left|\nabla f_{+}\right|^{2} d V_{s}-2 \int_{M} \varphi e^{g}<\nabla \varphi, \nabla f_{+}>f_{+} d V_{s} \\
& -\int_{M} \varphi^{2} e^{g} f_{+}<\nabla g, \nabla f_{+}>d V_{s} \\
\leq & 2 \int_{M} e^{g} f_{+}^{2}|\nabla \varphi|^{2} d V_{s}+\frac{1}{2} \int_{M} \varphi^{2} e^{g} f_{+}^{2}|\nabla g|^{2} d V_{s} . \quad \text { (1.12) }
\end{aligned}
$$

On the other hand,

$$
\begin{aligned}
& -\frac{1}{2} \int_{0}^{T} \int_{M} \varphi^{2} e^{g} \frac{\partial}{\partial t}\left(f_{+}^{2}\right) d V_{s} d s=\frac{1}{2}\left[-\left.\int_{M} \varphi^{2} e^{g} f_{+}^{2} d V_{s}\right|_{0} ^{T}\right. \\
& \left.\quad+\int_{0}^{T} \int_{M} \varphi^{2} e^{g} g_{s} f_{+}^{2} d V_{s} d s+\int_{0}^{T} \int_{M} \varphi^{2} e^{g} f_{+}^{2} F_{s}(y, s) d V_{s} d s\right] \\
& \leq \frac{1}{2}\left[-\left.\int_{M} \varphi^{2} e^{g} f_{+}^{2} d V_{s}\right|_{0} ^{T}+\int_{0}^{T} \int_{M} \varphi^{2} e^{g} g_{s} f_{+}^{2} d V_{s} d s\right]
\end{aligned}
$$

where we have used (1.8). Combining (1.10)-(1.13), we have that

$$
\int_{M} \varphi^{2}(x) e^{g(x, T)} f_{+}^{2}(x, T) d V_{T} \leq 4 \int_{0}^{T} \int_{M} e^{g} f_{+}^{2}|\nabla \varphi|^{2} d V_{s} d s .
$$

Now using (1.7) we have

$$
\int_{M} \varphi^{2}(x) e^{g(x, T)} f_{+}^{2}(x, T) d V_{T} \leq C_{3} \int_{0}^{T} \int_{M} e^{g} f_{+}^{2}|\widetilde{\nabla} \varphi|^{2} d V_{0} d s
$$

for some constant $C_{3}$ depending on $C_{1}$ in (1.7). Here $\widetilde{\nabla}$ is the gradient with respect the initial metric $g_{i j}(x, 0)$. For $R>0$, let $\varphi$ be the function with compact support such that

$$
\begin{aligned}
& \varphi(x)=1, \quad \text { for } \quad x \in B_{0}(o, R) \\
& \varphi(x)=0, \quad \text { for } \quad x \in M \backslash B_{0}(o, 2 R) ; \\
& |\widetilde{\nabla} \varphi| \leq \frac{2}{R} .
\end{aligned}
$$

Letting $R \rightarrow \infty$ in (1.14) we have that

$$
\int_{M} e^{g(x, T)} f_{+}^{2}(x, T) d V_{T} \leq \liminf _{R \rightarrow \infty} \frac{4 C_{3}}{R^{2}} \int_{0}^{T} \int_{B_{0}(o, 2 R) \backslash B_{0}(o, R)} e^{-\frac{r_{0}^{2}(x)}{C_{4} T}} f_{+}^{2} d V_{0} d s
$$


for some constant $C_{4}>0$ depending only on $C_{1}$ in (1.7). Now if $T<\frac{1}{a C_{4}}$, by (1.9), we will have

$$
\int_{M} e^{g(x, T)} f_{+}^{2}(x, T) d V_{T} \leq 0 .
$$

This implies that $f(x, T) \leq 0$. Since $C_{4}$ depends only on $C_{1}$, iterating this procedure we complete the proof of the theorem.

Let $g_{\alpha \bar{\beta}}(x, t)$ be a solution of $(1.1)$ on $M \times[0, T)$, which is Kähler for all $t$. We have the following easy lemma.

Lemma 1.1. Suppose there is a function $u_{0}(x)$ such that

$$
\sqrt{-1} \partial \bar{\partial} u_{0}=\operatorname{Ric}(g(\cdot, 0))
$$

where $\operatorname{Ric}(g(0))$ is the Ricci form of the initial metric $g(0)$. Let $F$ be the ratio of the volume element as in (1.5) and let $u(x, t)=u_{0}(x)-F(x, t)$. Then

$$
\begin{gathered}
\sqrt{-1} \partial \bar{\partial} u=\operatorname{Ric}(g(t)), \\
\left(\Delta-\frac{\partial}{\partial t}\right) u(x, t)=0, \\
\left(\Delta-\frac{\partial}{\partial t}\right)|\nabla u|^{2}=\left\|u_{\alpha \beta}\right\|^{2}+\left\|u_{\alpha \bar{\beta}}\right\|^{2}, \\
\left(\Delta-\frac{\partial}{\partial t}\right)\left(|\nabla u|^{2}+1\right)^{\frac{1}{2}} \geq 0,
\end{gathered}
$$

and

$$
\left(\Delta-\frac{\partial}{\partial t}\right) \mathcal{R}=\left(\Delta-\frac{\partial}{\partial t}\right) u_{t}=-\left\|u_{\alpha \bar{\beta}}\right\|^{2} .
$$

Here $\left\|u_{\alpha \bar{\beta}}\right\|^{2}(x, t)=g^{\alpha \bar{\beta}}(x, t) g^{\gamma \bar{\delta}}(x, t) u_{\alpha \bar{\delta}}(x, t) u_{\gamma \bar{\beta}}(x, t), \quad\left\|u_{\alpha \beta}\right\|^{2}(x, t)=$ $g^{\alpha \bar{\beta}}(x, t) g^{\gamma \bar{\delta}}(x, t) u_{\alpha \gamma}(x, t) u_{\bar{\beta} \bar{\delta}}(x, t)$.

Proof. (1.16) and (1.17) follow from the fact that $g_{\alpha \bar{\beta}}(x, t)$ is a solution of (1.1) which is Kähler, and the definition of $F$ and $u_{0}$.

To prove (1.18), after choosing a normal coordinates with respect to $g_{\alpha \bar{\beta}}(x, t)$ near any fixed point

$$
\begin{aligned}
\Delta|\nabla u|^{2} & =g^{\gamma \bar{\delta}}\left(u_{\alpha} u_{\bar{\beta}} g^{\alpha \bar{\beta}}\right)_{\gamma \bar{\delta}} \\
& =u_{\alpha \gamma} u_{\bar{\alpha} \bar{\gamma}}+u_{\alpha \bar{\gamma}} u_{\bar{\alpha} \gamma}+(\Delta u)_{\alpha} u_{\bar{\alpha}}+u_{\alpha}(\Delta u)_{\bar{\alpha}}+u_{\alpha \bar{\beta}} u_{\alpha} u_{\bar{\beta}},
\end{aligned}
$$


where we have used (1.1) and (1.16). Using (1.1), we have

$$
\frac{\partial}{\partial t}|\nabla u|^{2}=\left(u_{t}\right)_{\alpha} u_{\bar{\alpha}}+u_{\alpha}\left(u_{t}\right)_{\bar{\alpha}}+u_{\alpha \bar{\beta}} u_{a} u_{\bar{\beta}} .
$$

Combining this with (1.17), we have (1.18). (1.19) follows from (1.18) by direct computations.

To prove (1.20), differentiate (1.17) with respect to $t$. Using (1.16) we have

$$
\begin{aligned}
\left(\Delta-\frac{\partial}{\partial t}\right) \mathcal{R} & =\left(\Delta-\frac{\partial}{\partial t}\right) u_{t} \\
& =-g_{t}^{\alpha \bar{\beta}} u_{\alpha \bar{\beta}} \\
& =g^{\xi \bar{\beta}} g^{\alpha \bar{\gamma}} g_{\xi \bar{\gamma}, t} u_{\alpha \bar{\beta}} \\
& =-g^{\xi \bar{\beta}} g^{\alpha \bar{\gamma}} R_{\xi \bar{\gamma}} u_{\alpha \bar{\beta}} \\
& =-g^{\xi \bar{\beta}} g^{\alpha \bar{\gamma}} u_{\xi \bar{\gamma}} u_{\alpha \bar{\beta}} \\
& =-\left\|u_{\alpha \bar{\beta}}\right\|^{2} .
\end{aligned}
$$

This completes the proof of the lemma.

We are ready to prove the long time existence.

Theorem 1.3. Let $\left(M^{m}, g_{\alpha \bar{\beta}}(x, t)\right)$ be a complete noncompact Kähler manifold with nonnegative holomorphic bisectional curvature such that its scalar curvature $\mathcal{R}_{0}$ is bounded and satisfies

$$
\int_{0}^{\infty} k(x, s) d s \leq C_{1}
$$

for some constant $C_{1}$ for all $x$ and $r$, where

$$
k(x, s)=f_{B(x, s)} \mathcal{R}_{0} d V .
$$

Then (1.1) has long time existence. Moreover, there is a function $u(x, t)$ such that

$$
\begin{gathered}
\sqrt{-1} \partial \bar{\partial} u(\cdot, t)=\operatorname{Ric}(g(t)), \\
|\nabla u| \leq C(m) C_{1}
\end{gathered}
$$

and

$\mathcal{R}(x, t)+|\nabla u|^{2}(x, t) \leq \sup _{x \in M}\left(\mathcal{R}_{0}(x)+\left|\widetilde{\nabla} u_{0}\right|^{2}(x)\right) \leq \sup _{x \in M} \mathcal{R}_{0}(x)+\left(C(m) C_{1}\right)^{2}$ 
for some constant positive $C(m)$ depending only on $m$ and for all $(x, t)$. Moreover, the equality holds for some $\left(x_{0}, t_{0}\right)$, with $t_{0}>0$ if and only if $g_{\alpha \bar{\beta}}(x, t)$ is a Kähler-Ricci soliton.

Proof. By Theorem 1.1, there is a maximal $\infty \geq T_{\max }>0$ such that (1.1) has a solution $g_{\alpha \bar{\beta}}(x, t)$ which satisfies condition (i) in Theorem 1.1 for $0 \leq$ $t<T_{\max }$, and satisfies the following condition: For any $0<T<T_{\max }$, there is a constant $C>0$ such that (1.3) and (1.4) are true on $M \times[0, T]$. By (1.21) and the results in [N-S-T, Theorems 1.3 and 5.1], there is a function $u_{0}(x)$ such that

$$
\sqrt{-1} \partial \bar{\partial} u_{0}=\operatorname{Ric}(g(0))
$$

and

$$
\left|\widetilde{\nabla} u_{0}\right|(x) \leq C(m) C_{1}
$$

for all $x$ for some constant $C(m)$ depending only on $m$. Let $u(x, t)=u_{0}(x)-$ $F(x, t)$ and let $0<T<T_{\max }$ be fixed. By (1.3), (1.4), (1.6) and (1.25), it is easy to see that there is a constant $C_{2}$ such that for $(x, t) \in M \times[0, T]$

$$
|u(x, t)| \leq C_{2}\left(r_{0}(x)+1\right)
$$

where $r_{0}(x)$ is the distance from a fixed point $o$ with respect to $g(0)$. By Lemma 1.1 (1.16), we have $\Delta u(x, t)=\mathcal{R}(x, t)$. Combining this with (1.4) and (1.26), it is not hard to prove that

$$
\int_{B_{t}(o, r)}|\nabla u|^{2} \leq C_{3} r^{2 m+1}
$$

for some constant $C_{3}$ for all $0 \leq t \leq T$ and for all $r$. Here we have used the fact that $g_{\alpha \bar{\beta}}(x, t)$ has nonnegative Ricci curvature and volume comparison. Hence using (1.3), we conclude that the function $f=\left(|\nabla u|^{2}+1\right)^{\frac{1}{2}}-$ $\left(C^{2}(m) C_{1}^{2}+1\right)^{\frac{1}{2}}$ satisfies the condition (1.8) in Theorem 1.2 with $T_{1}$ replaced by $T$. Here $C(m)$ is the constant in (1.25). By (1.19) of Lemma 1.1 and Theorem 1.2, we can conclude that (1.23) is true for $x \in M$ and $0 \leq t \leq T_{\max }$, because $T$ can be any positive number less than $T_{\max }$.

By (1.18) and (1.20) of Lemma 1.1, we have

$$
\left(\Delta-\frac{\partial}{\partial t}\right)\left(|\nabla u|^{2}+\mathcal{R}\right)=\left\|u_{\alpha \beta}\right\|^{2} .
$$

By (1.23) and (1.4), we conclude that $|\nabla u|^{2}+\mathcal{R}$ is uniformly bounded on $M \times[0, T]$. By (1.28), we can apply Theorem 1.2 again and conclude that 
(1.24) is true for all $x \in M$ and $0 \leq t \leq T_{\max }$. In particular $\mathcal{R}$ is uniformly bounded on $M \times\left[0, T_{\max }\right)$. By Theorem 1.1, $T_{\max }$ must be infinity. If for some $\left(x_{0}, t_{0}\right), t_{0}>0$,

$$
\left(\mathcal{R}+|\nabla u|^{2}\right)\left(x_{0}, t_{0}\right)=\sup _{x \in M}\left(\mathcal{R}+|\nabla u|^{2}\right)(x, 0)
$$

we can conclude that $\mathcal{R}(x, t)+|\nabla u|^{2}(x, t)$ is constant, by the strong maximum principle. Thus $u_{\alpha \beta}(x, t)=0$ by (1.28). Together with the fact $u_{\alpha \bar{\beta}}(x, t)=$ $R_{\alpha \bar{\beta}}(x, t)$, it implies that $g_{\alpha \bar{\beta}}(x, t)$ is a Kähler-Ricci soliton. It is easy to check that for a Kähler Ricci soliton (1.24) holds with the equality (Cf. [C-H]).

\section{Some properties preserved by the Kähler-Ricci flow.}

In this section, we shall investigate the behavior of $f_{B_{t}\left(x_{0}, r\right)} \mathcal{R} d V_{t}$. To do this, we shall give some generalizations of the estimates in [Sh2-3, C-Z, C-T-Z] from above and below on the volume element $F(x, t)$ defined in (1.5). More precisely, we shall obtain upper and lower estimates on $F(x, t)$ in terms of the integral

$$
\int_{0}^{r} s k(x, s) d s
$$

where $k(x, s)$ is the average of the scalar curvature $\mathcal{R}_{0}$ over $B_{0}(x, s)$ at $t=0$. Our proofs use the well-known estimates of the heat kernels and the Green's functions for manifolds with nonnegative Ricci curvature of Li-Yau [L-Y]. Our proofs seem to be simpler than those in [Sh2-3], etc. Also we do not use the complicated construction of exhaustion functions as in the [Sh2-3, $\mathrm{C}-\mathrm{Z}, \mathrm{C}-\mathrm{T}-\mathrm{Z}]$. To derive our estimates we need the following lemma, which is a direct consequence of the mean value inequality of Li-Schoen [L-S] on subharmonic functions.

Lemma 2.1 (Generalized mean value inequality). Let $M^{n}$ be a complete noncompact Riemannian manifold with nonnegative Ricci curvature with real dimension $n$. Let $u \geq 0$ be a smooth function such that $\tilde{\Delta} u \geq-f$ with $f \geq 0$. For any $x_{0} \in M$ and $r>0$, we have

$$
u\left(x_{0}\right) \leq \int_{B\left(x_{0}, r\right)} G_{r}\left(x_{0}, y\right) f(y) d y+C(n) f_{B\left(x_{0}, r\right)} u
$$

for some constant $C(n)$ depending only on $n$, where $G_{r}(x, y)$ is the positive Green's function on $B\left(x_{0}, r\right)$ with zero boundary value. 
Proof. Let $v$ be such that $\tilde{\Delta} v=-f$ on $B\left(x_{0}, r\right)$ and $v=0$ on $\partial B\left(x_{0}, r\right)$. Note that $v \geq 0$ in $B\left(x_{0}, r\right)$. Since $w=\max \{u-v, 0\}$ is Lipschitz, subharmonic and nonnegative, by the mean value inequality of Li-Schoen [L-S], we have

$$
w\left(x_{0}\right) \leq C f_{B\left(x_{0}, r\right)} w
$$

for some constant $C=C(n)$ depending only on $n$. If $u\left(x_{0}\right)-v\left(x_{0}\right) \leq 0$, then we have

$$
u\left(x_{0}\right) \leq v\left(x_{0}\right)=\int_{B\left(x_{0}, r\right)} G_{r}\left(x_{0}, y\right) f(y) d y .
$$

In this case, $(2.1)$ is true. If $u\left(x_{0}\right)-v\left(x_{0}\right)>0$ then

$$
\begin{aligned}
u\left(x_{0}\right) & =w\left(x_{0}\right)+v\left(x_{0}\right) \\
& \leq C f_{B\left(x_{0}, r\right)} w+v\left(x_{0}\right) \\
& \leq C f_{B\left(x_{0}, r\right)} u+v\left(x_{0}\right) \\
& \leq C f_{B\left(x_{0}, r\right)} u+\int_{B\left(x_{0}, r\right)} G_{r}\left(x_{0}, y\right) f(y) d y .
\end{aligned}
$$

Therefore (2.1) is also true for this case.

We should mention that the above lemma was also proved in a somewhat different form in [Sh2-3] with a more complicated proof (Cf. Lemma 6.10 of [Sh2] and Lemma 6.8 of [Sh3]). We also need the following estimates of Green's functions.

Lemma 2.2. Let $M^{n}$ be as in Lemma 1.1. For any function $f \geq 0$, let $k(x, r)=f_{B(x, r)} f$. Then we have

$$
\int_{B(x, r)} G_{r}(x, y) f(y) d y \geq C(n)\left(r^{2} k\left(x, \frac{r}{5}\right)+\int_{0}^{\frac{r}{5}} s k(x, s) d r\right),
$$

for some constant $C(n)>0$ depending only on $n$, where $G_{r}$ is the Green's function on $B(x, r)$ where zero boundary value. If in addition, $M$ supports a minimal positive Green's function $G(x, y)$ such that

$$
\alpha \cdot \frac{r^{2}(x, y)}{V(x, r(x, y))} \leq G(x, y) \leq \frac{1}{\alpha} \cdot \frac{r^{2}(x, y)}{V(x, r(x, y))}
$$


for some $\alpha>0$ for all $x, y \in M$, then

$$
\int_{B(x, r)} G(x, y) f(y) d y \leq C(n, \alpha)\left(r^{2} k(x, r)+\int_{0}^{r} s k(x, s) d r\right),
$$

for some positive constant $C(n, \alpha)$ depending only on $n$ and $\alpha$.

Proof. See the proofs of [N-S-T, Theorems 1.1, 2.1].

In the rest of this section, we assume $M^{m}$ is a complete noncompact Kähler manifold with bounded nonnegative holomorphic bisectional curvature such that $g_{\alpha \bar{\beta}}$ is a solution of (1.1) on $M \times[0, T)$ with $T \leq \infty$. We also assume that conditions (i) and (ii) in Theorem 1.1 are satisfied by $g_{\alpha \bar{\beta}}$ on $M \times\left[0, T_{1}\right]$ for any $T_{1}<T$. Let $\mathfrak{m}(t)=\inf _{M} F(\cdot, t)$. Then $\mathfrak{m}(t) \leq 0$.

With the notations as in $\S 1$, we also need the following result of Shi [Sh3, p. 156].

\section{Lemma 2.3.}

$$
\begin{aligned}
\mathcal{R}_{0}(x) & \geq \mathcal{R}_{0}(x)+e^{F} F_{t} \\
& \geq \mathcal{R}_{0}(x)-g^{\alpha \bar{\beta}}(x, 0) R_{\alpha \bar{\beta}}(x, t) \\
& =\tilde{\Delta} F(x, t) \\
& \geq \mathcal{R}_{0}(x)-\mathcal{R}(x, t)
\end{aligned}
$$

where $\tilde{\Delta}$ is the Laplacian of the metric $g(0)$.

Theorem 2.1. With the above assumptions and notations, the following estimates are true. Namely there exists $C_{1}>0$ depending only on $m$ such that for all $\left(x_{0}, t\right) \in M \times[0, T)$

$$
-F\left(x_{0}, t\right) \geq C_{1}^{-1} \int_{0}^{\sqrt{t}} s k\left(x_{0}, s\right) d s
$$

and

$$
-F\left(x_{0}, t\right) \leq C_{1}\left[\left(1+\frac{t(1-\mathfrak{m}(t))}{R^{2}}\right) \int_{0}^{R} s k\left(x_{0}, s\right) d s-\frac{t \mathfrak{m}(t)(1-\mathfrak{m}(t))}{R^{2}}\right],
$$

where $k\left(x_{0}, t\right)=f_{B_{0}\left(x_{0}, r\right)} \mathcal{R}_{0} d V_{0}$. 
Proof. To prove (2.3), by Lemma 2.3 we have

$$
\tilde{\Delta} F \geq \mathcal{R}_{0}-\mathcal{R}=\mathcal{R}_{0}+F_{t}
$$

and so

$$
\left(\tilde{\Delta}-\frac{\partial}{\partial t}\right)(-F) \leq-\mathcal{R}_{0}
$$

Let $H(x, y, t)$ be the heat kernel of $M$ with respect to the metric $g(0)$, and let

$$
v(x, t)=\int_{0}^{t} \int_{M} H(x, y, t) \mathcal{R}_{0}(y) d V_{0}(y)
$$

Then $\tilde{\Delta} v-v_{t}=-\mathcal{R}_{0}$ and $v=0$ at $t=0$. By (2.5) and the fact that $F(\cdot, 0) \equiv 0$, by the maximum principle and the estimate of the heat kernel $[\mathrm{L}-\mathrm{Y}]$, we have for $(x, t) \in M \times[0, T)$

$$
\begin{aligned}
-F(x, t) & \geq v(x, t) \\
& =\int_{0}^{t} \int_{M}^{t} H(x, y, \tau) \mathcal{R}_{0}(y) d V_{0} d \tau \\
& \geq C_{2} \int_{0}^{t} \int_{0}^{\infty} \frac{1}{V_{0}(x, \sqrt{\tau})} e^{-\frac{r^{2}}{5 \tau}} \int_{\partial B_{0}(x, r)} \mathcal{R}_{0}(y) d A_{0} d r d \tau \\
& \geq C_{2} \int_{0}^{t} \int_{0}^{\sqrt{\tau}} \frac{1}{V_{0}(x, \sqrt{\tau})} e^{-\frac{r^{2}}{5 \tau}} \int_{\partial B_{0}(x, r)} \mathcal{R}_{0}(y) d A_{0} d r d \tau \\
& =C_{3} \int_{0}^{t} k(x, \sqrt{\tau}) d \tau \\
& =2 C_{3} \int_{0}^{\sqrt{t}} \tau k(x, \tau) d \tau .
\end{aligned}
$$

for some positive constants $C_{2}-C_{3}$ depending only on $m$. Hence (2.3) is true.

To prove (2.4), by Lemma 2.3, $\tilde{\Delta} F \leq \mathcal{R}_{0}+e^{F} F_{t}$. Hence for any $\left(x_{0}, t\right) \in$ $M \times[0, T)$ for any $R>0$, integrating the above inequality over $B_{0}\left(x_{0}, R\right) \times$ $[0, t]$, we have

$$
\begin{gathered}
\int_{0}^{t} \int_{B_{0}\left(x_{0}, R\right)} G_{R}\left(x_{0}, y\right) \tilde{\Delta} F(y, s) d V_{0} d s \\
\leq t \int_{B_{0}\left(x_{0}, R\right)} G_{R}\left(x_{0}, y\right) \mathcal{R}_{0}(y) d V_{0}+\int_{B_{0}\left(x_{0}, R\right)} G_{R}\left(x_{0}, y\right)\left(e^{F(y, t)}-1\right) d V_{0},
\end{gathered}
$$


and

$$
\begin{gathered}
\int_{B_{0}\left(x_{0}, R\right)} G_{R}\left(x_{0}, y\right)\left(1-e^{F(y, t)}\right) d V_{0} \\
\leq t \int_{B_{0}\left(x_{0}, R\right)} G_{R}\left(x_{0}, y\right) \mathcal{R}_{0}(y) d V_{0}+\int_{0}^{t} \int_{B_{0}\left(x_{0}, R\right)} G_{R}\left(x_{0}, y\right) \tilde{\Delta}(-F(y, s)) d V_{0} .
\end{gathered}
$$

By the Green's formula, for each $0 \leq s \leq t$

$$
\begin{aligned}
\int_{B_{0}\left(x_{0}, R\right)} G_{R}\left(x_{0}, y\right) \tilde{\Delta}(-F(y, s)) d V_{0} & =F\left(x_{0}, s\right)+\int_{\partial B_{0}\left(x_{0}, R\right)} F(y, s) \frac{\partial G_{R}\left(x_{0}, y\right)}{\partial \nu} \\
& \leq-\mathfrak{m}(t),
\end{aligned}
$$

where we have used the fact that $\mathfrak{m}(t)$ is nonincreasing, $F \leq 0$, $\frac{\partial}{\partial \nu} G_{R}\left(x_{0}, y\right) \leq 0$ and $\int_{\partial B_{0}\left(x_{0}, R\right)} \frac{\partial}{\partial \nu} G_{R}\left(x_{0}, y\right)=-1$. Combining this with (2.6), we have

$$
\int_{B_{0}\left(x_{0}, R\right)} G_{R}\left(x_{0}, y\right)\left(1-e^{F(y, t)}\right) d V_{0} \leq t\left(\int_{B_{0}\left(x_{0}, R\right)} G_{R}\left(x_{0}, y\right) \mathcal{R}_{0}(y) d V_{0}-\mathfrak{m}(t)\right) .
$$

Using the first inequality in Lemma 2.2, this implies

$$
R^{2} f_{B_{0}\left(x_{0}, \frac{1}{5} R\right)}\left(1-e^{F(y, t)}\right) d V_{0} \leq C_{4} t\left(\int_{B_{0}\left(x_{0}, R\right)} G_{R}\left(x_{0}, y\right) \mathcal{R}_{0}(y) d V_{0}-\mathfrak{m}(t)\right)
$$

for some constant $C_{4}$ depending only on $m$. Since if $0 \leq x \leq 1,1-e^{-x} \geq \frac{1}{3} x$, we have $\left(1-e^{F}\right)(1-\mathfrak{m}(t)) \geq-C F$ for some absolute positive constant $C$. Hence (2.7) implies that

$$
\begin{gathered}
R^{2} f_{B_{0}\left(x_{0}, \frac{1}{5} R\right)}(-F(y, t)) d V_{0} \\
\leq C_{5} t(1-\mathfrak{m}(t))\left(\int_{B_{0}\left(x_{0}, R\right)} G_{R}\left(x_{0}, y\right) \mathcal{R}_{0}(y) d V_{0}-\mathfrak{m}(t)\right)
\end{gathered}
$$

for some constant $C_{5}$ depending only on $m$. By Lemma $2.3, \tilde{\Delta}(-F) \geq-\mathcal{R}_{0}$. By Lemma 2.1 and (2.8), there is a constant $C_{6}$ depending only on $m$ such 
that

$$
\begin{aligned}
-F\left(x_{0}, t\right) \leq & \int_{B_{0}\left(x_{0}, \frac{1}{5} R\right)} G_{\frac{1}{5} R}\left(x_{0}, y\right) \mathcal{R}_{0}(y) d V_{0}+C(n) f_{B_{0}\left(x_{0}, \frac{1}{5} R\right)}(-F(y, t)) d V_{0} \\
\leq & \int_{B_{0}\left(x_{0}, \frac{1}{5} R\right)} G_{\frac{1}{5} R}\left(x_{0}, y\right) \mathcal{R}_{0}(y) d V_{0} \\
& +\frac{C_{6} t(1-\mathfrak{m}(t))}{R^{2}}\left(\int_{B_{0}\left(x_{0}, R\right)} G_{R}\left(x_{0}, y\right) \mathcal{R}_{0}(y) d V_{0}-\mathfrak{m}(t)\right), \quad(2.9)
\end{aligned}
$$

where $G_{\frac{1}{5} R}$ is the Green's function on $B_{0}\left(x_{0}, \frac{1}{5} R\right)$. As in [Sh3], by considering $M \times \mathbb{C}^{2}$, we may assume that $M$ has positive Green's function which satisfies the condition in Lemma 2.2. Applying Lemma 2.2, we can conclude from (2.9) that

$$
-F\left(x_{0}, t\right) \leq C_{7}\left[\left(1+\frac{t(1-\mathfrak{m}(t))}{R^{2}}\right) \int_{0}^{2 R} s k\left(x_{0}, s\right) d s-\frac{t \mathfrak{m}(t)(1-\mathfrak{m}(t))}{R^{2}}\right],
$$

for some constant $C_{7}$ depending only on $m$. This completes the proof of the theorem.

Corollary 2.1. Same assumptions and notations as in Theorem 2.1. Suppose $k(x, r) \leq k(r)$ for some function $k(r)$ for all $x \in M$. Then there exist positive constants $C$, a depending only on $m$ such that for $0 \leq t<T$

$$
-\mathfrak{m}(t) \leq C \int_{0}^{R} s k(s) d s
$$

where $R^{2}=a t(1-\mathfrak{m}(t))$.

Proof. By (2.4), we have for any $R>0$

$$
-\mathfrak{m}(t) \leq C_{1}\left[\left(1+\frac{t(1-\mathfrak{m}(t))}{R^{2}}\right) \int_{0}^{R} s k(s) d s-\frac{t \mathfrak{m}(t)(1-\mathfrak{m}(t))}{R^{2}}\right]
$$

where $C_{1}$ is a constant depending only on $m$. Let $R^{2}=2 C_{1} t(1-\mathfrak{m}(t))$, we have

$$
-\mathfrak{m}(t) \leq 2 C_{1}\left(1+\frac{1}{2 C_{1}}\right) \int_{0}^{R} s k(s) d s .
$$

From this the result follows. 
Corollary 2.2. With the same assumptions as in Corollary 2.1. Suppose

$$
\int_{0}^{r} s k(s) d s \leq r^{2} \phi(r)
$$

for all $r$, where $\phi(r)$ is a nonincreasing function of $r$ such that $\lim _{r \rightarrow \infty} \phi(r)=$ 0 . For $0<\tau \leq \sup \phi$, let

$$
\psi(\tau)=\sup \{r \mid \phi(r) \geq \tau\}
$$

Then for $0 \leq t<T$,

$$
-\mathfrak{m}(t) \leq \max \left\{1, \frac{C^{\prime}}{t} \psi^{2}\left(\frac{C^{\prime \prime}}{t}\right)\right\}
$$

for some positive constants $C^{\prime}$ and $C^{\prime \prime}$ depending only on $m$. In particular, the Kähler-Ricci flow has long time existence.

Proof. Note the $\psi(\tau)$ is finite and nonincreasing for $0<\tau \leq \sup \phi$ because $\phi(r) \rightarrow 0$ as $r \rightarrow \infty$. By Corollary 2.1, there exist constants $a$ and $C_{1}$ depending only on $m$ such that

$$
-\mathfrak{m}(t) \leq C_{1} \int_{0}^{R} s k(s) d s \leq C_{1} a t(1-\mathfrak{m}(t)) \phi(\sqrt{\operatorname{at}(1-\mathfrak{m}(t))})
$$

where $R^{2}=a t(1-\mathfrak{m}(t))$. Suppose $-\mathfrak{m}(t) \geq 1$, then the above inequality implies that

$$
\phi(\sqrt{a t(1-\mathfrak{m}(t))}) \geq \frac{1}{2 C_{1} a t} .
$$

In particular, $\frac{1}{2 C_{1} a t} \leq \sup \phi$. Hence

$$
\sqrt{a t(1-\mathfrak{m}(t))} \leq \psi\left(\frac{1}{2 C_{1} a t}\right)
$$

Hence

$$
-\mathfrak{m}(t) \leq \max \left\{1, \frac{C^{\prime}}{t} \psi^{2}\left(\frac{C^{\prime \prime}}{t}\right)\right\}
$$

for some positive constants $C^{\prime}$ and $C^{\prime \prime}$ depending only on $m$.

The last statement follows from the method in [Sh3, §7]. Here we cannot use the method in Theorem 1.3 because we do not have a good solution for the Poincaré-Lelong equation. 
Remark 2.1. The condition for long time existence in the corollary is weaker than that in [Sh3]. In [C-T-Z], the long time existence is proved for the case of surfaces under the assumptions that the surface has maximal volume growth and that $\int_{0}^{r} s k\left(x_{0}, s\right)=o\left(r^{2}\right)$. The last assumption is a little bit weaker than ours.

Remark 2.2. By the corollary, we may have the estimates in [Sh2-3]. For example, if $k(r)=C(1+r)^{-2}$, then it is easy to see that $-\mathfrak{m}(t) \leq C \log (t+1)$. If $k(r)=C(1+r)^{-\theta}$ for $0<\theta<2$, then $-\mathfrak{m}(t) \leq C(t+1)^{(2-\theta) / \theta}$. In addition to these results in [Sh2-3], we may have the following estimate. Namely, if $\int_{0}^{\infty} k(r) d r<\infty$, then $-\mathfrak{m}(t)=o(t)$ and if $\int_{0}^{r} s k(s) d s \leq C r^{2} / \log (2+r)$, then we have $-\mathfrak{m}(t) \leq e^{C t}$ for some $C>0$.

Another application of the corollary is a slight generalization of a gap theorem of Chen-Zhu [C-Z]. In [C-Z], it is proved that if $M$ is a complete Kähler manifold with bounded nonnegative holomorphic bisectional curvature such that

$$
k(x, r)=f_{B_{0}(x, r)} \mathcal{R}_{0} d V_{0} \leq \epsilon(r) r^{-2}
$$

for all $x$ and $r$, where $\epsilon(r) \rightarrow 0$ as $r \rightarrow \infty$. Then $M$ must be flat. Note that under this condition, the Kähler-Ricci flow has long time solution such that $R(x, t)$ is uniformly bounded on $M \times[0, \infty)$ by Theorem 1.3 and so $-\mathfrak{m}(t) \leq C t$. Moreover

$$
\int_{0}^{r} s k(x, s) d s=o(\log r)
$$

uniformly.

Using Corollary 2.1, we have:

Corollary 2.3. Let $\left(M^{m}, g\right)$ be complete Kähler manifold with bounded nonnegative holomorphic bisectional curvature such that the Kähler-Ricci flow (1.1) has long time solution.

(a) Suppose $M$ is nonflat and $-\mathfrak{m}(t) \leq C t^{k}$ for some constant $C$ and $k>0$. Then

$$
\begin{gathered}
\liminf _{r \rightarrow \infty} \frac{\int_{0}^{r} s k(x, s) d s}{\log r}>0, \\
\liminf _{t \rightarrow \infty} \frac{-F(x, t)}{\log t}>0
\end{gathered}
$$


and

$$
\liminf _{t \rightarrow \infty} t \mathcal{R}(x, t)>0
$$

for all $x$, where $k(x, s)=f_{B_{0}(x, r)} \mathcal{R}_{0} d V_{0}$.

(b) If the Poisson equation $\tilde{\Delta} u=\mathcal{R}_{0}$ has a solution $u$ which is bounded from above, then $M$ is flat. In particular, any bounded from above solution is a constant.

Proof. Note that if (2.11) is true for some $x$, it is true for all $x$. Suppose $M$ is nonflat, then there exists $x_{0}$ such that $\mathcal{R}_{0}\left(x_{0}\right)>0$. If $(2.11)$ is not true, then there exists $R_{i} \rightarrow \infty$ such that

$$
\int_{0}^{R_{i}} s k\left(x_{0}, s\right) d s \leq \frac{1}{i} \log R_{i} .
$$

Let $t_{i} \rightarrow \infty$ be such that $t_{i}\left(1-\left(\mathfrak{m}\left(t_{i}\right)\right)^{2}=R_{i}^{2}\right.$. By (2.4), we have

$$
\begin{aligned}
-F\left(x_{0}, t_{i}\right) & \leq C_{1}\left(\int_{0}^{R_{i}} s k\left(x_{0}, s\right) d s+1\right) \\
& \leq C_{1}\left(\frac{1}{i} \log R_{i}+1\right) \\
& \leq C_{2}\left(\frac{1}{i} \log t_{i}+1\right)
\end{aligned}
$$

for some constants $C_{1}-C_{2}$ independent of $i$. Here we have used the assumption that $-\mathfrak{m}(t) \leq C t^{k}$. We can then proceed as in [C-Z]. For any $T>0$, by the Li-Yau-Hamilton type inequality [Co2-3] for $t>T$,

$$
\frac{T}{t} \mathcal{R}\left(x_{0}, T\right) \leq \mathcal{R}\left(x_{0}, t\right) .
$$

Integrating from $T$ to $t_{i}$, we have

$$
T \log \frac{t_{i}}{T} \mathcal{R}\left(x_{0}, T\right) \leq-F\left(x_{0}, t_{i}\right) \leq C_{2}\left(\frac{1}{i} \log t_{i}+1\right) .
$$

Dividing both sides by $\log t_{i}$ and let $t_{i} \rightarrow \infty$, we have $\mathcal{R}\left(x_{0}, T\right)=0$. Since $T$ is arbitrary, we conclude that $\mathcal{R}\left(x_{0}\right)=0$. This is a contradiction. Hence (2.11) is true.

If $(2.12)$ is not true for some $x$, then by (2.3) in Theorem 2.1, (2.11) is not true for this $x$. Hence $M$ must be flat by the previous result. 
By (2.12), for any $x \in M$ there exists $C_{3}>0$ and $t_{0}>0$ such that

$$
-F(x, t) \geq C_{3} \log t,
$$

for all $t \geq t_{0}$. By the Li-Yau-Hamilton type inequality in [Co2-3], for all $t>t_{0}$ and $s \leq t$,

$$
\frac{t}{s} \mathcal{R}(x, t) \geq \mathcal{R}(x, s) .
$$

Integrating over $s$ from 1 to $t$ and using (2.16) we have

$$
\begin{aligned}
(t \log t) \mathcal{R}(x, t) & \geq \int_{1}^{t} \mathcal{R}(x, s) d s \\
& =-F(x, t)-\int_{0}^{1} \mathcal{R}(x, s) d s \\
& \geq C_{3} \log t-\int_{0}^{1} \mathcal{R}(x, s) d s .
\end{aligned}
$$

From this (2.13) follows.

The proof of (b) follows from the proof of (a) and Theorem 2.1 of [N-S-T].

Remark 2.3. The argument above in fact also shows that any bounded solution to $\tilde{\Delta} u=\mathcal{R}_{0}(x)$ is a constant since if $\tilde{\Delta} u=\mathcal{R}_{0}(x)$ has a bounded solution, we then have long time solution to (0.2) by Theorem 1.3 and Theorem 2.1 of $[\mathrm{N}-\mathrm{S}-\mathrm{T}]$. In $[\mathrm{CW}$, a gradient estimate is obtained for the Kähler-Ricci flow under the assumption that there is a bounded potential function for the Ricci tensor. If we assume the manifold has nonnegative holomorphic bisectional curvature, then this is only possible for flat manifolds.

Corollary 2.4. Same assumptions and notations as in Corollary 2.1. If we assume that

$$
\lim _{r \rightarrow \infty} \frac{\int_{0}^{r} s k(s) d s}{r}=0
$$

we have long time existence for the Kähler-Ricci flow with

$$
\lim _{t \rightarrow \infty} \frac{-\mathfrak{m}(t)}{t}=0
$$

and

$$
\lim _{t \rightarrow \infty} R(x, t)=0
$$

uniformly for $x \in M$. If in addition, we assume that $(M, g(0))$ has maximum volume growth, $M$ is diffeomorphic to $\mathbb{R}^{2 m}$, in case $m \geq 3$ and homeomorphic to $\mathbb{R}^{4}$, in case $m=2$. Moreover, $M$ is a Stein manifold. 
Proof. The first part just follows from Corollary 2.1 and the Li-Yau-Hamilton type inequality of Cao [Co2-3] as in the proof of Corollary 2.3. To prove that $M$ is Stein and topologically $\mathbb{R}^{2 m}$ one just need to use the observation that the injectivity radius of $M$ has a uniform lower bound in the case of the maximum volume growth and bounded curvature tensor. Also $|R(x, t)| \rightarrow 0$, as $t \rightarrow \infty$, means that the Kähler-Ricci flow will improves the injectivity radius to $\infty$ along the flow. The rest argument is same as in section 3 of [C-Z].

Another corollary of the proof of Theorem 2.1 is a result on the preservation of volume growth under the Kähler-Ricci flow. In [Sh2] it was proved that the property of having maximum volume growth is preserved under the assumption that $\mathcal{R}_{0}(x)$ is of quadratic decay. In [C-Z, C-T-Z] it was generalized to the case of more relaxed decay conditions on $\mathcal{R}_{0}(x)$ using the same argument as [Sh2]. In [H3], it was proved under the Ricci flow with nonnegative Ricci curvature, and under the stronger assumption that the Riemannian curvature tensor of the initial metric goes to zero pointwisely, then the volume ratio $\lim _{r \rightarrow \infty} r^{-n} V_{t}(r)$ is preserved. In our case, we have the following stronger result:

Theorem 2.2. With the same assumptions and notations as in Theorem 2.1. Suppose for some $x_{0}$,

$$
\int_{0}^{r} s k\left(x_{0}, s\right) d s=o\left(r^{2}\right) \quad \text { as } r \rightarrow \infty .
$$

Let $o \in M$ be a fixed point. Then for any $0<t<T$,

$$
\lim _{r \rightarrow \infty} \frac{V_{t}(o, r)}{V_{0}(o, r)}=1
$$

where $V_{t}(o, r)$ is the volume of the geodesic ball $B_{t}(o, r)$ with respect to the metric $g(t)$ for $0 \leq t<T$.

Proof. Since $\mathcal{R}(x, t)$ is uniformly bounded on $M \times[0, t]$, by Theorem 17.2 in [H3], $B_{t}(o, r) \subset B_{0}\left(o, r+C_{1} t\right)$ for some constant $C_{1}$ independent of $r$. Using the fact that $g(t)$ is nonincreasing in $t$, we have that

$$
\begin{aligned}
V_{t}(o, r) & \leq V_{t}\left(B_{0}\left(o, r+C_{1} t\right)\right) \\
& \leq V_{0}\left(B_{0}\left(o, r+C_{1} t\right)\right) \\
& \leq V_{0}(o, r) \cdot\left(\frac{r+C_{1} t}{r}\right)^{2 m} .
\end{aligned}
$$


This implies that

$$
\limsup _{r \rightarrow \infty} \frac{V_{t}(o, r)}{V_{0}(o, r)} \leq 1 .
$$

Using the fact that $g(t)$ is nonincreasing in $t$ again, we have

$$
\begin{aligned}
V_{t}(o, r) & \geq \int_{B_{0}(o, r)} d V_{t} \\
& =\int_{B_{0}(o, r)} e^{F(y, t)} d V_{0} \\
& =V_{0}(o, r)+\int_{B_{0}(o, r)}\left(e^{F(y, t)}-1\right) d V_{0} .
\end{aligned}
$$

On the other hand, using (2.7) in the proof of Theorem 2.1 and using Lemma 2.2 as in the proof of (2.4), we have

$$
f_{B_{0}(o, r)}\left(1-e^{F(y, t)}\right) d V_{0} \leq C_{2} r^{-2} t\left(\int_{0}^{10 r} s k(o, s) d s-\mathfrak{m}(t)\right)
$$

for some constant $C_{2}$ independent on $r$. Combining this with (2.17), we have

$$
\frac{V_{t}(o, r)}{V_{0}(o, r)} \geq 1-C_{2} r^{-2} t\left(\int_{0}^{10 r} s k(o, s) d s-\mathfrak{m}(t)\right) .
$$

Since $\int_{0}^{R} s k(s) d s=o\left(R^{2}\right)$, we have

$$
\liminf _{r \rightarrow \infty} \frac{V_{t}(o, r)}{V_{0}(o, r)} \geq 1
$$

The theorem then follows.

It was proved in [H3] that the condition $|R m| \rightarrow 0$ as $x \rightarrow \infty$ is preserved under the Ricci flow. Applying Theorem 2.1, we can prove that the decay rate of the scalar curvature in the average sense is preserved under the Kähler-Ricci flow in a certain sense.

Theorem 2.3. Let $M^{m}$ be a complete noncompact Kähler manifold with bounded nonnegative holomorphic bisectional curvature. Suppose (1.1) has long time existence, such that for any $T>0$ the conditions (i) and (ii) in Theorem 1.1 are satisfied. Then the following are true:

(a) Suppose $\int_{0}^{r} s k(x, s) d s \leq C(1+r)^{1-\epsilon}$ for some constants $C>0$ and $\epsilon>0$ for all $x$ and $r$. Then $\int_{0}^{r} s k_{t}(x, s) d s \leq C^{\prime}(1+r)^{\delta}$ where $\delta=$ $\min \{1,2(1-\epsilon) /(1+\epsilon)\}$ for some constant $C^{\prime}$ independent of $x, t, r$. 
(b) Suppose $\int_{0}^{r} s k(x, s) d s \leq C \log (r+2)$ for some constants $C>0$ for all $x$ and $r$. Then $\int_{0}^{r} s k_{t}(x, s) d s \leq C^{\prime} \log (r+2)$ for some constant $C^{\prime}$ independent of $x, t, r$.

Here $k(x, r)=f_{B_{0}(x, r)} \mathcal{R}_{0} d V_{0}$ and $k_{t}(x, r)=f_{B_{t}(x, r)} \mathcal{R}(y, t) d V_{t}$.

Proof. We prove (b) first. For $T \geq 0$, let

$$
F(x, t ; T)=\log \left[\frac{\operatorname{det}\left(g_{\alpha \bar{\beta}}(x, t+T)\right)}{\operatorname{det}\left(g_{\alpha \bar{\beta}}(x, T)\right)}\right] .
$$

Considering the flow $g_{\alpha \bar{\beta}}(x, t+T)$ with initial data $g_{\alpha \bar{\beta}}(x, T)$ and using $(2.3)$ in Theorem 2.1, we have for any $t>0$

$$
-F(x, t ; T) \geq C_{1} \int_{0}^{\sqrt{t}} s k_{T}(x, s) d s .
$$

for some constant $C_{1}>0$ depending only on $m$. On the other hand, by the Li-Yau-Hamilton inequality [Co2-3]

$$
T \mathcal{R}(x, T) \leq t \mathcal{R}(x, t)
$$

for all $t \geq T$. We have

$$
\int_{T}^{t} \frac{T}{s} \mathcal{R}(x, T) d s \leq \int_{T}^{t} \mathcal{R}(x, s) d s \leq-F(x, t ; 0) \leq C_{2} \log (t+2)
$$

for some constant $C_{2}$ independent of $x$ and $t$, where we have used Corollary 2.1 and the assumption on $k(x, r)$. Dividing both sides by $\log t$ and let $t \rightarrow \infty$, using the fact that $\mathcal{R}$ is uniformly bounded on $M \times[0, \infty)$ by Theorem 1.3, we have

$$
\mathcal{R}(x, T) \leq \frac{C_{3}}{T+1}
$$

for some constant $C_{3}$ independent of $x$ and $t$. Since the metric is nonincreasing along the Ricci flow $\operatorname{det}\left(g_{\alpha \bar{\beta}}(x, T)\right) \leq \operatorname{det}\left(g_{\alpha \bar{\beta}}(x, 0)\right)$, by $(2.18)$ and Theorem 2.1, for all $t>0$

$$
\begin{aligned}
\log (t+T+2) & \geq-C_{4} F(x, t+T ; 0) \\
& \geq-C_{4} F(x, t ; T) \\
& \geq C_{5} \int_{0}^{\sqrt{t}} s k_{T}(x, s) d s
\end{aligned}
$$


for some positive constants $C_{4}-C_{5}$ independent of $x, t$ and $T$. Suppose $r^{2} \geq T$, then we take $t=r^{2}$ in (2.20), we have

$$
\int_{0}^{r} s k_{T}(x, s) d s \leq C_{6} \log (r+2)
$$

for some constant $C_{6}$ independent of $x, t, T$. Suppose $r^{2} \leq T$, then by (2.19), we have

$$
\int_{0}^{r} s k_{T}(x, s) d s \leq C_{3} \frac{r^{2}}{T+1} \leq C_{7} \log (r+2)
$$

where $C_{7}$ is a constant independent of $x, t, T$. (b) follows from (2.21) and (2.22).

To prove (a), if $2(1-\epsilon) /(1+\epsilon)<1$, the proof is similar to the proof of (b). If $2(1-\epsilon) /(1+\epsilon) \geq 1$, the assumption in (a) implies that $\int_{0}^{\infty} k(x, s) d s \leq C_{8}$ for all $r$ and for all $x$. By Theorem 1.3, for any $t$ we can solve the PoincaréLelong equation $\sqrt{-1} \partial \bar{\partial} u=\operatorname{Ric}(g(t))$ with $|\nabla u|(x, t) \leq C_{9}$ for some constant independent of $x$ and $t$. By Theorem 2.1 in [N-S-T], the result follows.

\section{Asymptotic behavior of the volume element.}

In $\S 2$, we gave some estimates of the volume element $-F(x, t)$ under the Kähler-Ricci flow. In general, $-F(x, t)$ has no limit as $t \rightarrow \infty$ unless the original manifold is flat. In this section, we will use the Poincaré-Lelong equation and the results in $[\mathrm{N}-\mathrm{S}-\mathrm{T}]$ to obtain information on asymptotic behavior of the rescaled volume element $-F(x, t)+F\left(x_{0}, t\right)$. Let us assume that $\left(M^{m}, g_{\alpha \bar{\beta}}(x)\right)$ is a complete noncompact Kähler manifold with bounded nonnegative holomorphic bisectional curvature. As before, denote

$$
k(x, r)=f_{B(x, r)} \mathcal{R}_{0} d V_{0}
$$

where $\mathcal{R}_{0}$ is the scalar curvature of $g_{\alpha \bar{\beta}}$. We also assume that

$$
k(x, r) \leq k(r)
$$

for all $x \in M$, with $\int_{0}^{\infty} k(r) d r<\infty$. By Theorem 1.3, (1.1) has a long time solution $g_{\alpha \bar{\beta}}(x, t)$ with $g_{\alpha \bar{\beta}}(x, 0)=g_{\alpha \bar{\beta}}(x)$. On the other hand, by the result in $[\mathrm{N}-\mathrm{S}-\mathrm{T}]$, there is a unique function $u$ such that

$$
\sqrt{-1} \partial \bar{\partial} u_{0}=\operatorname{Ric}(g(0))
$$

with $u_{0}(o)=0$ and $\left|u_{0}\right|=o(r)$. We have the following: 
Theorem 3.1. Let $x_{0} \in M$ be a fixed point. For any $t_{j} \rightarrow \infty$, there is a subsequence, which is also denoted by $t_{j}$, such that

$$
\lim _{j \rightarrow \infty}\left(F\left(x, t_{j}\right)-F\left(x_{0}, t_{j}\right)\right)=u_{0}(x)-u_{0}\left(x_{0}\right)-v(x)
$$

where $u_{0}$ is the function in (3.2) and $v(x)$ is a pluriharmonic function of at most linear growth (with respect to the initial metric). The convergence is uniform on compact sets. If in addition, $\int_{0}^{r} s k(x, s) d s \leq C(1+r)^{1-\epsilon}$ with $\epsilon>1 / 3$, then

$$
\lim _{t \rightarrow \infty}\left(F(x, t)-F\left(x_{0}, t\right)\right)=u_{0}(x)-u_{0}\left(x_{0}\right)
$$

and the convergence is uniform on compact sets of $M$.

Proof. Let $h(x, t)=\left(u_{0}(x)-F(x, t)\right)-\left(u_{0}\left(x_{0}\right)-F\left(x_{0}, t\right)\right)$. By Theorem 1.3, there exists a constant $C_{1}$ such that for all $(x, t) \in M \times[0, \infty)$

$$
|\widetilde{\nabla} h(x, t)| \leq|\nabla h(x, t)| \leq C_{1},
$$

where $\widetilde{\nabla} h$ is the gradient with respect to the initial metric $g(0)$, and we have used the fact that $g_{\alpha \bar{\beta}}$ is nonincreasing. Since $h\left(x_{0}, t\right)=0$ for all $t$, it is easy to see that for any $t_{j} \rightarrow \infty$, there is a subsequence, which will be denoted by $t_{j}$ again, such that

$$
\lim _{j \rightarrow \infty} h\left(x, t_{j}\right)=v(x)
$$

for some Lipschitz continuous function $v(x)$ on $M$ with bounded gradient. Since

$$
\tilde{\Delta} h(x, t)=g^{\alpha \bar{\beta}}(x, 0) R_{\alpha \bar{\beta}}(x, t)
$$

for all $x$, where $\tilde{\Delta}$ is the Laplacian with respect to $g(0)$,

$$
0 \leq g^{\alpha \bar{\beta}}(x, 0) R_{\alpha \bar{\beta}}(x, t) \leq g^{\alpha \bar{\beta}}(x, t) R_{\alpha \bar{\beta}}(x, t)=\mathcal{R}(x, t)
$$

Since by Corollary $2.4, \lim _{t \rightarrow \infty} \mathcal{R}(x, t)=0$ uniformly on $M$, we conclude that $v(x)$ is a harmonic function of at most linear growth. Notice that $h(x, t)$ is plurisubharmonic. Thus $v$ is also plurisubharmonic. Together with the fact that it is also harmonic, $v$ must be pluriharmonic.

Suppose $\int_{0}^{r} s k(x, s) d s \leq C(1+r)^{1-\epsilon}$ with $\epsilon>1 / 3$. Then by Theorem 2.3 , we have

$$
\int_{0}^{r} s k_{t}(x, s) d s \leq C_{2}(1+r)^{\delta}
$$


for some constant $C_{2}>0$ independent of $x$ and $t$. Here $k_{t}(x, s)=$ $f_{B_{t}(x, s)} \mathcal{R} d V_{t}$ and $\delta=2(1-\epsilon) /(1+\epsilon)<1$. By Theorem 1.2 in [N-S-T] and the fact that $h(x, t)=o\left(r_{t}\left(x, x_{0}\right)\right)$ for fixed $t$, we can conclude from (3.5) that

$$
h(x, t) \leq C_{3}\left(1+r_{t}\left(x, x_{0}\right)\right)^{\delta} \leq C_{3}\left(1+r_{0}\left(x, x_{0}\right)\right)^{\delta}
$$

for some constant independent of $t$. Hence the harmonic function $v(x)$ is of sublinear growth and must be constant by [C-Y]. Since $v\left(x_{0}\right)=0, v$ must be identically zero.

In [Sh2] and later in [C-Z], it was proved that if $M$ is a complete noncompact Kähler manifold with positive and bounded holomorphic bisectional curvature such that the scalar curvature satisfies $f_{B(x, r)} \mathcal{R}_{0} \leq k(r)$ for all $x$ and $r$ with with $k(r) \leq C(1+r)^{-1-\epsilon}, \epsilon>1 / 2$, then the long time solution of the Kähler-Ricci flow subconverges after rescaling in the following sense. Let $x_{0}$ be a fixed point in $M$ and let $v$ be a fixed $(1,0)$ vector at $x_{0}$ with unit length with respect to the initial metric. Let $\widehat{g}_{\alpha \bar{\beta}}(x, t)=g_{\alpha \bar{\beta}}(x, t) / g_{v \bar{v}}\left(x_{0}, t\right)$. Then for any $t_{j} \rightarrow \infty$, we can find a subsequence, also denoted by $t_{j}$, such that $\widehat{g}_{\alpha \bar{\beta}}\left(x, t_{j}\right)$ converge uniformly on compact sets of $M$ to a flat Kähler metric. (There is a gap in their proofs, see the footnote on p. 114.). However, as pointed out in [C-Z], it is unclear whether the metric is complete. Using Theorem 3.1, we can get some preliminary estimates for the limiting metric.

Proposition 3.1. Let $\left(M^{m}, g_{\alpha \bar{\beta}}\right)$ be a complete noncompact Kähler manifold with positive and bounded holomorphic bisectional curvature such that the scalar curvature $\mathcal{R}_{0}$ satisfies

$$
f_{B(x, r)} \mathcal{R}_{0} d V_{0} \leq k(r)
$$

for all $x$ and $r$, where $k(r) \leq C(1+r)^{-1-\epsilon}$ with $\epsilon>1 / 2$. Let $g_{\alpha \bar{\beta}}(x, t)$ be the long time solution of (1.1) with $g_{\alpha \bar{\beta}}(x, 0)=g_{\alpha \bar{\beta}}(x)$. Suppose $\widehat{g}_{\alpha \bar{\beta}}\left(x_{0}, t\right)$ is equivalent to $g_{\alpha \bar{\beta}}\left(x_{0}, 0\right)$, uniform in $t$.

(a) The rescaled metrics

$$
\tilde{g}_{\alpha \bar{\beta}}(x, t)=e^{-\frac{F\left(x_{0}, t\right)}{m}} g_{\alpha \bar{\beta}}(x, t)
$$

subconverge to a flat Kähler metric $h_{\alpha \bar{\beta}}$ on $M$. The convergence is uniform on compact sets, where $x_{0}$ is a fixed point and

$$
F(x, t)=\log \frac{\operatorname{det}\left(g_{\alpha \bar{\beta}}(x, t)\right)}{\operatorname{det}\left(g_{\alpha \bar{\beta}}(x, 0)\right)} .
$$


(b) If, in addition, $\epsilon=1$ and $\mathcal{R}_{0}(x) \leq C r_{0}^{-2}(x)$, where $r_{0}(x)$ is the distance function from $x_{0}$ with respect to the initial metric, then

$$
\frac{\operatorname{det}\left(h_{\alpha \bar{\beta}}(x)\right)}{\operatorname{det}\left(g_{\alpha \bar{\beta}}(x, 0)\right)} \geq C^{\prime} r_{0}^{a}(x)-C^{\prime \prime}
$$

for some positive constants $a, C^{\prime}$ and $C^{\prime \prime}$. In particular, the maximal eigenvalue $\lambda_{\max }(x)$ of $h_{\alpha \bar{\beta}}(x)$ with respect to $g_{\alpha \bar{\beta}}(x, 0)$ satisfies

$$
\lambda_{\max }(x) \geq C^{\prime \prime \prime} r_{0}^{\frac{a}{m}}(x)
$$

for some positive constant $C^{\prime \prime \prime}$, provided $r_{0}(x)$ is large enough.

Proof. Part (a) follows from the method in [Sh2], see also [C-Z]. Since

$$
\log \frac{\operatorname{det}\left(h_{\alpha \bar{\beta}}(x, t)\right)}{\operatorname{det}\left(g_{\alpha \bar{\beta}}(x, 0)\right)}=\lim _{t \rightarrow \infty}\left(F(x, t)-F\left(x_{0}, t\right)\right),
$$

by Theorem 3.1 we have

$$
\log \frac{\operatorname{det}\left(h_{\alpha \bar{\beta}}(x, t)\right)}{\operatorname{det}\left(g_{\alpha \bar{\beta}}(x, 0)\right)}=u(x)-u\left(x_{0}\right)
$$

where $u(x)$ is the solution for the Poincaré-Lelong equation obtained in [N-S-T, Theorem 5.1]. Since $M$ is nonflat, by Remark 2.2 and Corollary 2.3 , we have

$$
\liminf _{r \rightarrow \infty} \frac{\int_{0}^{r} s k\left(x_{0}, s\right) d s}{\log r}>0 .
$$

By [N-S-T, Corollary 1.1], (3.8) and (3.9), we conclude that (3.6) is true.

(3.7) follows from (3.6) immediately.

\section{References.}

[Co1] H.-D. Cao, Deformation of Kähler metrics to Kähler-Einstien metrics on compact Kähler manifolds, Invent. Math. 81 (1985), 359372 .

[Co2] H.-D. Cao, On Harnack inequalities for the Kähler-Ricci flow, Invent. Math. 109 (1992), 247-263.

[Co3] H.-D. Cao, Limits of solutions to the Kähler-Ricci flow, J. Differential Geom. 45 (1997), 257-272. 
[C-H] H.-D. Cao and R. Hamilton, Gradient Kähler-Ricci solitons and periodic orbits, Comm. Anal. Geom. 8 (2000) no. 3, 517-529.

[C-T] A. Chau and L.-F. Tam, Gradient Kähler-Ricci solitons and a uniformization conjecture, preprint.

[C-Z] B. L. Chen and X. P. Zhu, On complete noncompact Kähler manifolds with positive bisectional curvature, preprint.

[C-T-Z] B. L. Chen, S. H. Tang and X. P. Zhu, A uniformization theorem of complete noncompact Kähler surfaces with positive bisectional curvature, preprint.

[C-Y] S. Y. Cheng and S.-T. Yau, Differential equations on Riemannian manifolds and their geometric applications, Comm. Pure Appl. Math. 28 (1975), 333-354.

[Cw] B. Chow, A gradient estimate for the Ricci-Kähler flow, Ann. Global Anal. Geom. 19 (2001), 321-325.

[H1] R. S. Hamilton, Three-manifolds with positive Ricci curvature, J. Differential Geom. 17 (1982), 255-306.

[H2] R. S. Hamilton, Four-manifolds with positive curvature operator, J. Differential Geom. 24 (1986), 153-179.

[H3] R. S. Hamilton, Formation of singularities in the Ricci flow, Surveys in Differential Geom. 2 (1995), 7-136.

[H4] R. S. Hamilton, The Harnack estimate for the Ricci flow, J. Differential Geom. 37 (1993), 225-243.

[K-L] K. Karp and P. Li, The heat equation on complete Riemannian manifolds, unpublished, 1982.

[Li] P. Li, Lecture notes on heat equations on complete Riemannian manifolds, Lectures at UCI, 1991.

[L-S] P. Li and R. Schoen, $L^{p}$ and mean value properties of subharmonic functions on Riemannian manifolds, Acta Math. 153 (1984), 279301.

[L-T] G. Liao and L.-F. Tam, On the heat equation for harmonic maps from non-compact manifolds, Pacific J. Math. 153 (1992), 129-145. 
[L-Y] P. Li and S.-T. Yau, On the parabolic kernel of the Schrödinger operator, Acta Math. 156 (1986), 139-168.

[M] K. Mok, The uniformization theorem for compact Kähler manifolds of nonnegative holomorphic bisectional curvature, J. Differential Geom. 27 (1988), 179-214.

[M-S-Y] N. Mok, Y.-T. Siu and S.-T. Yau, The Poincaré-Lelong equation on complete Kähler manifolds, Compositio Math. 44 (1981), 183-218.

[N1] L. Ni, Vanishing theorems on complete Kähler manifolds and their applications, J. Differential Geom. 50 (1998), 89-122.

[N2] L. Ni, Poisson equation and Hermitian-Einstein metrics on holomorphic vector bundles over complete noncompact Kähler manifolds, Indiana Univ. Math. Jour. 51 (2002), 670-703.

[N-S-T] L. Ni, Y.-G. Shi and L.-F. Tam, Poisson equation, Poincaré-Lelong equation and curvature decay on complete Kähler manifolds, J. Differential Geom. 57 (2001), 339-388.

[Sh1] W. X. Shi, Deforming the metric on complete Riemannian manifolds, J. Differential Geom. 30 (1989), 223-301.

[Sh2] W. X. Shi, Ricci deformation of metric on complete noncompact Kähler manifolds, Ph. D. thesis Harvard University, 1990.

[Sh3] W. X. Shi, Ricci flow and the uniformization on complete noncompact Kähler manifolds, J. Differential Geom. 45 (1997), 94-220.

[Sh4] W. X. Shi, A uniformization theorem for complete Kähler manifolds with positive holomorphic bisectional curvature, Jour. Geom. Analysis 8 (1998), 117-142.

[Y1] S.-T. Yau, Harmonic functions on complete Riemannian manifolds, Comm. Pure Appl. Math. 28 (1975), 201-228.

[Y2] S.-T. Yau, On the Ricci curvature of a compact Kähler manifolds and the complex Monge-Amperé equation I., Comm. Pure Appl. Math. 31 (1978), 339-411.

\section{LEI NI}

Department of Mathematics

University of California, San Diego 
LA Jolla, CA 92093

lni@math.ucsd.edu

LUEN-FAI TAM

DePartment of Mathematics

The Chinese University of Hong Kong

Shatin, Hong Kong, China

lftammath. cuhk. edu.hk 
L. Ni and L.-F. Tam 